\title{
New perspective for the magnetic corrections to $\pi$ - $\pi$ scattering lengths in the linear sigma model
}

\author{
M. Loewe, ${ }^{1,2,3, *}$ L. Monje, ${ }^{1, \dagger}$ E. Muñoz, ${ }^{1, \$}$ A. Raya, ${ }^{4, \S}$ and R. Zamora ${ }^{5,6, \|}$ \\ ${ }^{1}$ Instituto de Física, Pontificia Universidad Católica de Chile, Casilla 306, Santiago 22, Chile \\ ${ }^{2}$ Centre for Theoretical and Mathematical Physics and Department of Physics, University of Cape Town, \\ Rondebosch 7700, South Africa \\ ${ }^{3}$ Centro Científico Tecnológico de Valparaíso-CCTVAL, Universidad Técnica Federico Santa María, \\ Casilla 110-V, Valparaíso, Chile \\ ${ }^{4}$ Instituto de Física y Matemáticas, Universidad Michoacana de San Nicolás de Hidalgo, Edificio C-3, \\ Ciudad Universitaria, Morelia 58040, Michoacán, México \\ ${ }^{5}$ Instituto de Ciencias Básicas, Universidad Diego Portales, Casilla 298-V, Santiago, Chile \\ ${ }^{6}$ Centro de Investigación y Desarrollo de Ciencias Aeroespaciales (CIDCA), Fuerza Aérea de Chile, \\ Santiago 8020744, Chile
}

(Received 13 January 2019; published 6 March 2019)

\begin{abstract}
In this article, a new perspective for obtaining the magnetic evolution of $\pi$ - $\pi$ scattering lengths in the linear sigma model is presented. When computing the relevant one-loop diagrams that contribute to these parameters, the sum over Landau levels-emerging from the expansion of the Schwinger propagator-is handled in a novel way that could also be applied to the calculation of other magnetic-type corrections. Essentially, we obtain an expansion in terms of Hurwitz zeta functions. It is necessary to regularize our expressions by an appropriate physical subtraction when $|q B| \rightarrow 0$ (where $q$ is the meson charge and $B$ is the magnetic field strength). In this way, we are able to interpolate between the very high-magnetic-fieldstrength region (usually handled in terms of the lowest Landau level approximation) and the weak-field region (discussed in a previous paper by some of us), which is based on an appropriate expansion of the Schwinger propagator up to order $|q B|^{2}$. Our results for the scattering length parameters produce a soft evolution for a wide range of magnetic field strengths, reducing to the previously found expressions in both limits.
\end{abstract}

DOI: $10.1103 /$ PhysRevD.99.056002

\section{INTRODUCTION}

During recent years, the physics of strongly interacting hadron matter at high temperatures and densities (or baryonic chemical potential), including the huge magnetic fields generated during peripheral heavy-ion collisions, has attracted the attention of the community, from both the experimental and theoretical points of view. Different experiments have reported interesting results that are related to temperature and eventually to magnetic corrections of some physical quantities. For example, an excess of

\footnotetext{
*mloewe@fis.puc.cl

†nmonje@uc.cl

*munozt@fis.puc.cl

\$raya@ifm.umich.mx

rzamorajofre@gmail.com
}

Published by the American Physical Society under the terms of the Creative Commons Attribution 4.0 International license. Further distribution of this work must maintain attribution to the author(s) and the published article's title, journal citation, and DOI. Funded by SCOAP ${ }^{3}$. photons at low momentum in the invariant momentum distribution has been reported [1-3]. It has been argued [4] that this excess-after taking into account common sources like synchrotron radiation, bremsstrahlung, or pair annihilation - could be related to gluon fusion in the early stages of the collision. This mechanism is only possible if magnetic effects are present. Signals related only to temperature effects are much more well established such as, e.g., the broadening of hadron resonances [5]. Of course, the effect on the broadening of resonance widths should take into account simultaneous corrections of the temperature and magnetic field.

New experiments like NICA [6] would be able to explore density effects, allowing the experimental discussion of scenarios like quarkyonic matter [7]. Of course, a dense nuclear matter environment is one of the crucial ingredients for our understanding of compact objects like neutron stars. Lattice groups [8] have also found the remarkable phenomenon of inverse magnetic catalysis, which corresponds to a decrease of the pseudocritical temperatures of both the chiral and/or deconfinement 
phase transitions and the quark condensates, as functions of an increasing magnetic field strength. Different explanations have been proposed, including, e.g., an analysis that went beyond the mean-field approximation by considering thermomagnetic corrections to the couplings as well as plasma screening for the boson masses through ring diagrams in the linear sigma model [9]. It is worth mentioning that, as a consequence of a renormalization group analysis, an analytical expression for the thermomagnetic evolution of the QCD strong coupling was recently found [10]. This should allow to extend existing theoretical calculations.

It seems, therefore, that the discussion of the magnetic dependence of different physical parameters is highly relevant for our understanding of these kind of physical phenomena. In general, it is not an easy task to disentangle magnetic effects from other kinds of corrections, and this represents an important motivation to search for new channels or parameters where this goal could be achieved. $\pi-\pi$ scattering lengths are responsible for the interaction among pions at low energies, near the threshold. Since about 600 pions are produced in a single heavy-ion collision event, and since charmonium and bottomonium states-which may survive beyond the critical temperature-decay into pairs of pions whose rescattering has been measured, we see that scattering lengths could be relevant parameters for a better understanding of magnetic and thermal evolution. In fact, the so called cusp effect in the emerging pions from such heavy onium states has been used as a clear signal for measuring $\pi-\pi$ scattering lengths [11]. It is certainly a challenge to measure such signals in heavy-ion collision experiments. The new results we present here are no longer restricted to the low-magnetic field regime, as was the case in a previous article by some of us [12].

Some years ago, analyses were done on the temperature dependence of these scattering length parameters using the Nambu-Jona-Lasinio [13] and linear sigma models [14]. As previously mentioned, magnetic effects on these objects were computed in the linear sigma model by some of us using an expansion of the Schwinger propagator valid for small magnetic fields [12]. The main result of that articlenamely, the opposite effect of the magnetic field and temperature-was interesting, since it seems that magnetic and temperature effects have opposite behavior. For the isospin $I=0,2$ channels, the $\pi$ - $\pi$ scattering lengths increase or decrease as a function of temperature. The opposite effects were found for the magnetic evolution.

Here we present, in the linear sigma model at the oneloop level, a new discussion on the magnetic dependence of the $\pi-\pi$ scattering lengths, valid for arbitrary values of the magnetic field strength. The novelty of the analysis relies on the way we handle the relevant integrals that appear in the one-loop diagrams. In fact, using the wellknown expansion for the Schwinger propagator in terms of Landau levels, and introducing a physically transparent regularization of a certain magnetic-field-dependent logarithmic divergent term, we are able to obtain rather compact expansions for the relevant one-loop integrals associated with the $s$ - and $t$-channel contributions. The paper is organized as follows. In Sec. II the linear sigma model is revised, and we decompose the $\pi-\pi$ scattering lengths according to isospin channel projections. Then, in Sec. III the detailed computation of the magnetic field contribution to the $\pi-\pi$ scattering lengths is presented, including the regularization of a magnetic-dependent divergent term. In this way, we are able to present our results for the magnetic evolution for the scattering lengths in both relevant isospin channels $I=0,2$. More technical details are presented in the Appendix. Finally, in Sec. IV we present our final conclusions.

\section{LINEAR SIGMA MODEL AND $\pi$ - $\pi$ SCATTERING}

The linear sigma model was introduced by Gell-Mann and Lévy [15] as an effective approach to describe chiral symmetry breaking via an explicit and spontaneous mechanism. In the context of critical phenomena, the model represents a field theory where the Lagrangian possesses $O(N)$ symmetry, which near the critical temperature is spontaneously broken into $O(N-1)$, thus leading to $N-1$ massless Goldstone bosons (representing tangential oscillating modes) and a single massive field (representing radial oscillations) with respect to the minimum of a Mexican-hat-shaped effective potential.

In the phase where the chiral symmetry is broken, the model is given by

$$
\begin{aligned}
\mathcal{L}= & \bar{\psi}\left[i \gamma^{\mu} \partial_{\mu}-m_{\psi}-g\left(s+i \vec{\pi} \cdot \vec{\tau} \gamma_{5}\right)\right] \psi \\
& +\frac{1}{2}\left[(\partial \vec{\pi})^{2}+m_{\pi}^{2} \vec{\pi}^{2}\right]+\frac{1}{2}\left[(\partial \sigma)^{2}+m_{\sigma}^{2} s^{2}\right] \\
& -\lambda^{2} v s\left(s^{2}+\vec{\pi}^{2}\right)-\frac{\lambda^{2}}{4}\left(s^{2}+\vec{\pi}^{2}\right)^{2}+\left(\varepsilon c-v m_{\pi}^{2}\right) s .
\end{aligned}
$$

In this expression $v=\langle\sigma\rangle$ is the vacuum expectation value of the scalar field $\sigma$. The idea is to define a new field $s$ such that $\sigma=s+v$, with $\langle s\rangle=0 . \psi$ corresponds to an isospin doublet associated to the nucleons, $\vec{\pi}$ denotes the pion isotriplet field, and $c \sigma$ is the term that explicitly breaks the $S U(2) \times S U(2)$ chiral symmetry. $\varepsilon$ is a small dimensionless parameter. It is interesting to remark that all fields in the model have masses determined by $v$. In fact, the following relations are valid: $m_{\psi}=g v, m_{\pi}^{2}=\mu^{2}+\lambda^{2} v^{2}$, and $m_{\sigma}^{2}=$ $\mu^{2}+3 \lambda^{2} v^{2}$. Perturbation theory at the tree level allows us to identify the pion decay constants as $f_{\pi}=v$. Finite-temperature effects on this model have been studied by several authors, including the thermal evolution of masses, $f_{\pi}(T)$, the effective potential, etc. [16-23]. 
Since our idea is to use the linear sigma model to calculate $\pi-\pi$ scattering lengths, let us briefly recall the formalism. A scattering amplitude has the general form $[24,25]$

$$
\begin{aligned}
T_{\alpha \beta ; \delta \gamma}= & A(s, t, u) \delta_{\alpha \beta} \delta_{\delta \gamma}+A(t, s, u) \delta_{\alpha \gamma} \delta_{\beta \delta} \\
& +A(u, t, s) \delta_{\alpha \delta} \delta_{\beta \gamma},
\end{aligned}
$$

where $\alpha, \beta, \gamma, \delta$ denote isospin components.

By using appropriate projection operators, it is possible to find the following isospin-dependent scattering amplitudes:

$$
\begin{gathered}
T^{0}=3 A(s, t, u)+A(t, s, u)+A(u, t, s), \\
T^{1}=A(t, s, u)-A(u, t, s), \\
T^{2}=A(t, s, u)+A(u, t, s),
\end{gathered}
$$

where $T^{I}$ denotes a scattering amplitude in a given isospin channel $I=\{0,1,2\}$.

As it is well known [24], the isospin-dependent scattering amplitude can be expanded in partial waves $T_{\ell}^{I}$,

$$
T_{\ell}^{I}(s)=\frac{1}{64 \pi} \int_{-1}^{1} d(\cos \theta) P_{\ell}(\cos \theta) T^{I}(s, t, u) .
$$

Below the inelastic threshold, the partial scattering amplitudes can be parametrized as [25]

$$
T_{\ell}^{I}=\left(\frac{s}{s-4 m \pi^{2}}\right)^{\frac{1}{2}} \frac{1}{2 i}\left(e^{2 i \delta_{\ell}^{I}(s)}-1\right),
$$

where $\delta_{\ell}$ is a phase shift in the $\ell$ channel. The scattering lengths are important parameters for describing low-energy interactions. In fact, the last expression can be expanded according to

$$
\Re\left(T_{\ell}^{I}\right)=\left(\frac{p^{2}}{m_{\pi}^{2}}\right)^{\ell}\left(a_{\ell}^{I}+\frac{p^{2}}{m_{\pi}^{2}} b_{\ell}^{I}+\cdots\right) .
$$

The parameters $a_{\ell}^{I}$ and $b_{\ell}^{I}$ are the scattering lengths and scattering slopes, respectively. In general, the scattering lengths obey $\left|a_{0}^{I}\right|>\left|a_{1}^{I}\right|>\left|a_{2}^{I}\right| \ldots$ If we are only interested in the scattering lengths $a_{0}^{I}$, it is enough to calculate the scattering amplitude $T^{I}$ in the static limit, i.e., when $s \rightarrow 4 m_{\pi}^{2}, t \rightarrow 0$, and $u \rightarrow 0$ :

$$
a_{0}^{I}=\frac{1}{32 \pi} T^{I}\left(s \rightarrow 4 m_{\pi}^{2}, t \rightarrow 0, u \rightarrow 0\right) .
$$

The first measurement of $\pi-\pi$ scattering lengths was carried out by Rosellet et al. [26]. More recently, these parameters have been measured using pionium atoms in the DIRAC experiment [27] and also through the decay of heavy quarkonium states into $\pi$ - $\pi$ final states where the so-called cusp effect was found [28]. We evaluate Eq. (9) for $I=0,2$ in a background magnetic field of arbitrary strength below.

\section{SCATTERING LENGTHS AT FINITE MAGNETIC FIELD}

Recently, some of us discussed the magnetic evolution of the $\pi-\pi$ scattering lengths in the context of the linear sigma model [12].

Our analysis was based on a perturbative treatment of the bosonic Schwinger propagator, valid for small magnetic fields. We found that this magnetic evolution displays an opposite trend with respect to thermal corrections to the scattering lengths reported previously in the literature [14]. At low magnetic field intensities, the scattering lengths in the isospin channel $I=2$ increase, whereas their projections into the channel $I=0$ decrease (both as functions of the magnetic field). It is interesting to reanalyze this problem over the full range of magnetic field intensities. In fact, in peripheral heavy-ion collisions we may expect extremely high magnetic fields, which may affect the interactions among the emerging pions generated during the collision.

In the linear sigma model, the relevant diagrams that contribute to $\pi-\pi$ scattering are shown in Fig. 1. Notice that tadpole-like diagrams associated with the mass corrections of the sigma field do not contribute to the $\pi-\pi$ scattering amplitudes, because they do not possess an absorptive component, since their imaginary part is zero.
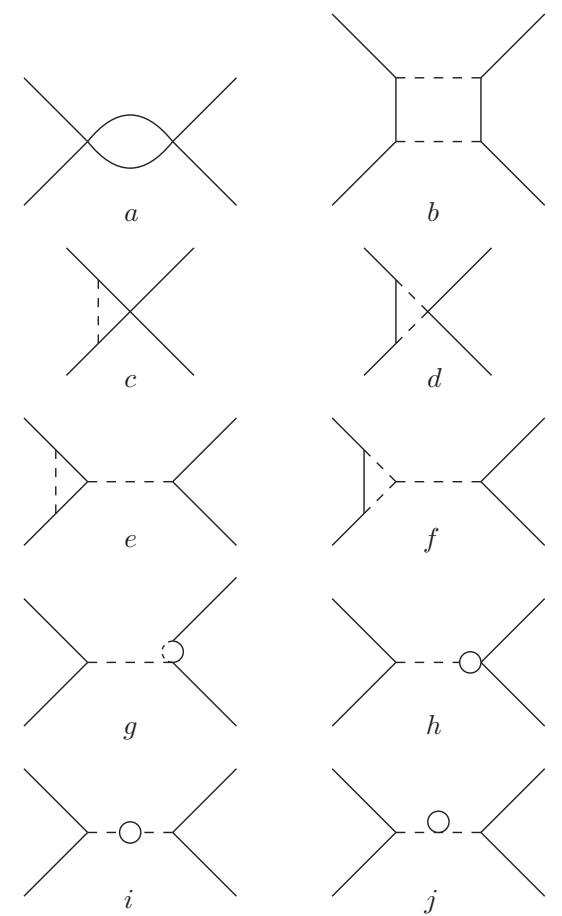

FIG. 1. One-loop diagrams relevant to the $\pi-\pi$ scattering lengths. Solid and dashed lines represent pions and $\sigma$ mesons, respectively. 


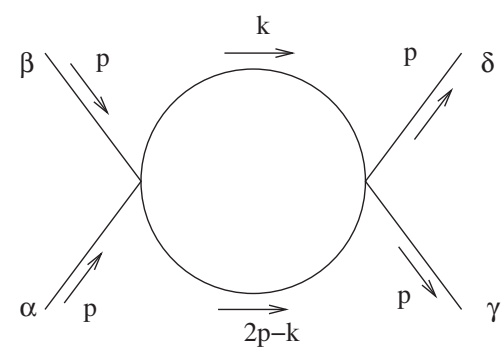

(a) s-channel diagram.

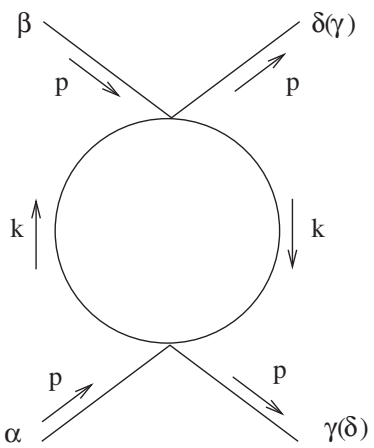

(b) $t$ and $u$ channel diagram.

FIG. 2. "Fish-type" diagrams.

These tadpoles are extremely small in the limit of a very large mass of the sigma field. This approximation is valid since, as we know, $m_{\sigma} \approx 550 \mathrm{MeV}$ is much larger than the pion mass. Fermions, i.e., nucleons that may interact with pions, are not considered in our discussion. As a consequence, the sigma field propagator is contracted to a point.

From these considerations, we see that all relevant diagrams reduce to horizontal ( $s$ channel) or vertical ( $t$ and $u$ channels) "fish-type" pion loop contributions, as shown in Fig. 2. Then, our task is to compute such diagrams as a function of the magnetic field intensity. This is an interesting problem, not only because of its physical implications, but also due to new analytical results that we present below.

Let us derive our starting expression for the bosonic propagator as a sum of Landau levels. The bosonic Schwinger propagator for a charged pion of charge $q$ immersed in a uniform magnetic field along the third spatial coordinate in the proper time representation is given by

$$
i D^{B}(k)=\int_{0}^{\infty} \frac{d s}{\cos (q B s)} e^{i s\left(k_{\|}^{2}-k_{\perp}^{2} \frac{\tan (q B s)}{q B s}-m_{\pi}^{2}+i \epsilon\right)} .
$$

After inserting this propagator into the fish-type diagrams, it is not difficult to see that all contributions reduce to two types of integrals:

$I_{1}\left[B, p_{0}\right]=\int \frac{d^{4} k}{(2 \pi)^{4}} i D^{B}\left(k_{0}, \mathbf{k}\right) i D^{B}\left(k_{0}-2 p_{0}, \mathbf{k}\right)$,

$$
I_{2}[B]=\int \frac{d^{4} k}{(2 \pi)^{4}}\left[i D^{B}\left(k_{0}, \mathbf{k}\right)\right]^{2} .
$$

For technical purposes, we shall calculate the integrals with the expression for the propagator at finite magnetic field in terms of Landau levels, as presented in Ref. [29],

$$
i D^{B}(k)=2 \sum_{l=0}^{\infty}(-1)^{l} L_{l}\left(\frac{2 k_{\perp}^{2}}{q B}\right) e^{-k_{\perp}^{2} / q B} i \Delta_{l}^{B}\left(k_{\|}\right),
$$

where $L_{l}(z)$ are the Laguerre polynomials, and we have defined the effective "parallel" propagators

$$
i \Delta_{l}^{B}\left(k_{\|}\right)=\frac{i}{k_{\|}^{2}-(2 l+1) q B-m_{\pi}^{2}+i \epsilon} .
$$

Let us first consider the calculation of $I_{1}[B]$ : given its definition in Eq. (11), by substituting the infinite series for the propagators (13) we have

$$
\begin{aligned}
I_{1}\left[B, p_{0}\right]= & \int \frac{d^{2} k_{\|} d^{2} k_{\perp}}{(2 \pi)^{4}} i D^{B}(k) i D^{B}\left(k_{0}-2 p_{0}, \mathbf{k}\right) \\
= & 4 \sum_{l=0}^{\infty} \sum_{l^{\prime}=0}^{\infty}(-1)^{l+l^{\prime}} G_{l, l^{\prime}}\left(p_{0}\right) \\
& \times\left[\int \frac{d^{2} k_{\perp}}{(2 \pi)^{2}} e^{-2 k_{\perp}^{2} / q B} L_{l}\left(\frac{2 k_{\perp}^{2}}{q B}\right) L_{l^{\prime}}\left(\frac{2 k_{\perp}^{2}}{q B}\right)\right] .
\end{aligned}
$$

Here, we have defined the integrals

$$
G_{l, l^{\prime}}\left(p_{0}\right)=\int \frac{d^{2} k_{\|}}{(2 \pi)^{2}} i \Delta_{l}^{B}\left(k_{\|}\right) i \Delta_{l^{\prime}}^{B}\left(k_{\|}-2 p_{0}\right) .
$$

Let us now calculate the integral over the Laguerre polynomials in the second term by using two-dimensional "spherical coordinates," with $0 \leq\left|k_{\perp}\right|<\infty$,

$$
d^{2} k_{\perp}=2 \pi\left|k_{\perp}\right| d\left|k_{\perp}\right|=\frac{\pi q B}{2} d x,
$$

where we have defined the auxiliary variable $x=2 k_{\perp}^{2} / q B$, with $0 \leq x<\infty$. Therefore, we have

$$
\begin{aligned}
& \int \frac{d^{2} k_{\perp}}{(2 \pi)^{2}} e^{-2 k_{\perp}^{2} / q B} L_{l}\left(\frac{2 k_{\perp}^{2}}{q B}\right) L_{l^{\prime}}\left(\frac{2 k_{\perp}^{2}}{q B}\right) \\
& =\frac{1}{4 \pi^{2}} \frac{\pi q B}{2} \int_{0}^{\infty} d x e^{-x} L_{l}(x) L_{l^{\prime}}(x) \\
& =\frac{q B}{8 \pi} \delta_{l, l^{\prime}},
\end{aligned}
$$

where the orthogonality relation between Laguerre polynomials was used. Substituting this result into Eq. (16), we end up with the expression 


$$
I_{1}\left[B, p_{0}\right]=\frac{q B}{2 \pi} \sum_{l=0}^{\infty} G_{l, l}\left(p_{0}\right)
$$

As shown in detail in the Appendix, we calculate $G_{l, l}\left(p_{0}\right)$ by first integrating over $k_{0}$ in the complex plane, and later over $k_{3}$. This procedure allows us to obtain the infinite series

$$
I_{1}\left[B, p_{0}\right]=\frac{i}{16 \pi^{2}} \frac{2 q B}{p_{0}^{2}} \sum_{l=0}^{\infty} z_{l} \operatorname{Arctan}\left(z_{l}\right),
$$

where we have defined $z_{l}=\left(p_{0} / \sqrt{2 q B}\right) /$ $\sqrt{l+1 / 2+\left(m_{\pi}^{2}-p_{0}^{2}\right) /(2 q B)}$. This infinite series, as expected, displays a mild logarithmic divergence, which can however be removed with a straightforward procedure, as we now show. Let us first expand each term in the series above using the infinite series (valid for $\left|z_{l}\right|>1$ and $\left.\left|z_{l}\right|<1\right)$

$$
\begin{aligned}
z_{l} \operatorname{Arctan}\left(z_{l}\right) & =\sum_{m=0}^{\infty} \frac{2^{2 m}(m !)^{2}}{(2 m+1) !}\left(\frac{z_{l}^{2}}{1+z_{l}^{2}}\right)^{m+1} \\
& =\sum_{m=0}^{\infty} \frac{2^{2 m}(m !)^{2}}{(2 m+1) !}\left(1+z_{l}^{-2}\right)^{-m-1} .
\end{aligned}
$$

Inserting Eq. (21) back into Eq. (20), and exchanging the order of the sums, we obtain

$$
\begin{aligned}
I_{1}\left[B, p_{0}\right]= & \frac{i}{16 \pi^{2}} \sum_{m=0}^{\infty} \frac{2^{2 m}(m !)^{2}}{(2 m+1) !}\left(\frac{p_{0}^{2}}{2 q B}\right)^{m} \\
& \times \zeta\left(1+m, \frac{1}{2}+\frac{m_{\pi}^{2}}{2 q B}\right),
\end{aligned}
$$

where $\zeta(\alpha, z)=\sum_{l=0}^{\infty}(z+l)^{-\alpha}$ are the Hurwitz zeta functions. It is important to remark that the term $m=0$ needs to be regularized, using the relation between the Hurwitz zeta function and the digamma function $\psi(z)$,

$$
\zeta(z, 1+\epsilon)=-\psi(z)+\frac{1}{\epsilon}+O(\epsilon), \quad \epsilon \rightarrow 0^{+} .
$$

The asymptotic behavior of the digamma function for very large values of its argument $(|z| \gg 1)$ is captured by the series

$$
\psi(z) \sim \ln (z)-\sum_{n=1}^{\infty} \frac{B_{n}}{n} z^{-n},
$$

where $B_{k}$ are the Bernouilli numbers, for $B_{1}=1 / 2$. Clearly, the digamma function displays a logarithmic divergence in this limit. Therefore, the expression for $I_{1}[B]$ in Eq. (22) diverges as $B \rightarrow 0$, as expected from the vacuum contribution to the diagram at zero field. Since we are interested in the contribution due to the finite magnetic field with respect to the experimental zero-field value of the scattering length, we define the regularized expression

$$
\begin{aligned}
I_{1}^{\mathrm{Reg}}\left[B, p_{0}\right] \equiv & I_{1}\left[B, p_{0}\right]-I_{1}\left[B \rightarrow 0, p_{0}\right] \\
= & \int \frac{d^{4} k}{(2 \pi)^{4}}\left[i D^{B}\left(k_{0}, \mathbf{k}\right) i D^{B}\left(k_{0}-2 p_{0}, \mathbf{k}\right)\right. \\
& \left.-i D^{0}\left(k_{0}, \mathbf{k}\right) i D^{0}\left(k_{0}-2 p_{0}, \mathbf{k}\right)\right],
\end{aligned}
$$

where clearly, by definition,

$$
\lim _{B \rightarrow 0} I_{1}^{\mathrm{Reg}}\left[B, p_{0}\right]=0
$$

In order to construct the regularized form, we subtract the asymptotic, logarithmically divergent expression for the digamma function $(m=0)$ at small magnetic field as follows:

$$
\begin{aligned}
I_{1}^{\operatorname{Reg}}\left[B, p_{0}\right]= & \frac{i}{16 \pi^{2}}\left[-\psi\left(\frac{1}{2}+\frac{m_{\pi}^{2}}{2 q B}\right)+\ln \left(\frac{1}{2}+\frac{m_{\pi}^{2}}{2 q B}\right)\right. \\
& +\sum_{m=1}^{\infty} \frac{(m !)^{2}}{(2 m+1) !}\left(2 \frac{p_{0}^{2}}{q B}\right)^{m} \\
& \left.\times \zeta\left(m+1, \frac{1}{2}+\frac{m_{\pi}^{2}}{2 q B}\right)\right] .
\end{aligned}
$$

Let us now turn our attention to the integral $I_{2}[B]$ defined in Eq. (12). It is straightforward to obtain the regularized expression for this integral by setting $p_{0}=0$ as follows:

$$
\begin{aligned}
I_{2}^{\mathrm{Reg}}[B] & \equiv I_{2}[B]-I_{2}[B \rightarrow 0] \\
& =\lim _{p_{0} \rightarrow 0} I_{1}^{\mathrm{Reg}}\left[B, p_{0}\right] \\
& =\frac{i}{16 \pi^{2}}\left[-\psi\left(\frac{1}{2}+\frac{m_{\pi}^{2}}{2 q B}\right)+\ln \left(\frac{1}{2}+\frac{m_{\pi}^{2}}{2 q B}\right)\right] .
\end{aligned}
$$

In order to obtain the scattering lengths $a_{0}^{I}$, we use the decomposition of the scattering amplitude in the different isospin channels presented in Sec. I. Since we are only interested in the scattering lengths $a_{0}^{I}$, it is enough to calculate the scattering amplitude in the static limit. Therefore, we normalize by the experimental values at tree level $\left[a_{0}^{0}(\exp )=0.217\right.$ and $\left.a_{0}^{2}(\exp )=-0.041\right]$ to obtain the expressions 


$$
\begin{aligned}
a_{0}^{0}(B)= & a_{0}^{0}(\exp )+\frac{1}{32 \pi}(3 A(s, t, u)+A(t, s, u) \\
& +A(u, t, s)), \\
a_{0}^{2}(B)= & a_{0}^{2}(\exp )+\frac{1}{32 \pi}(A(t, s, u)+A(u, t, s)) .
\end{aligned}
$$

Here, $A(s, t, u), A(t, s, u)$, and $A(u, t, s)$ correspond to all $s$-channel, $t$-channel, and $u$-channel contributions, respectively. On the other hand, the $s$-channel contribution is obtained from $I_{1}^{\mathrm{Reg}}\left[B, p_{0}=m_{\pi}\right]$, while those for the $t$ and $u$ channels are obtained from $I_{2}^{\mathrm{Reg}}[B]$ according to the following expressions:

$$
\begin{aligned}
A(s, t, u)= & -4 \lambda^{4}\left(1-\frac{12 \lambda^{2} v^{2}}{m_{\sigma}^{2}}+\frac{24 \lambda^{4} v^{4}}{m_{\sigma}^{4}}\right) \\
& \times I_{1}^{\operatorname{Reg}}\left[B, p_{0}=m_{\pi}\right], \\
A(t, s, u)+A(u, t, s)= & -8 \lambda^{4}\left(1-\frac{12 \lambda^{2} v^{2}}{m_{\sigma}^{2}}+\frac{24 \lambda^{4} v^{4}}{m_{\sigma}^{4}}\right) \\
& \times I_{2}^{\operatorname{Reg}}[B] .
\end{aligned}
$$

The experimental values in the absence of a magnetic field $(B=0)$ are given by [30] $a_{0}^{0}(\exp )=0.217$ and $a_{0}^{2}(\exp )=-0.041$. The mass for the sigma meson is set to $m_{\sigma}=550 \mathrm{MeV}$, and the mass for the pion $m_{\pi}=$ $140 \mathrm{MeV}$, with the parameters $v=89$ and $\lambda^{2}=4.26$.

\section{RESULTS AND CONCLUSIONS}

We have presented a novel method of calculating the scattering lengths for $\pi-\pi$ scattering within the linear sigma model at the one-loop level, in the isospin channels $I=\{0,2\}$, as functions of the external magnetic field intensity. Our calculation shows that the relevant contributions can be reduced to the calculation of two types of

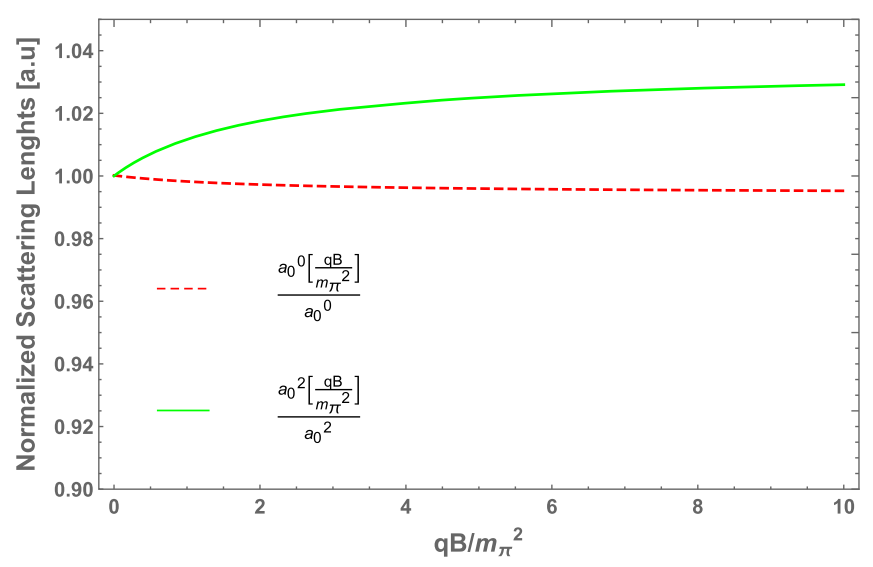

FIG. 3. The scattering parameters $a_{0}^{0}$ (dashed) and $a_{0}^{2}$ (solid) are displayed as functions of the dimensionless magnetic field $q B / m_{\pi}^{2}$. In the figure, the parameters are normalized by their (experimental) zero-field values. "fish-type" diagrams (see Fig. 2). Throughout this article, we have obtained exact analytical results for the integrals involved in those diagrams and, moreover, we developed a regularization procedure that allows to connect smoothly and continuously the low and high magnetic field intensity regimes. Explicit analytical expressions for the regularized integrals are presented in Eqs. (27) and (29), respectively. This method extends our previous results [12] to the full range of magnetic field intensities, thus revealing that the scattering lengths are smooth and continuous functions of the field (see Fig. 3). In particular, our analytical results show that the scattering length $a_{0}^{0}$ decreases as a function of the magnetic field with respect to its experimental value. On the contrary, the scattering length $a_{0}^{2}$ is a monotonically increasing function (in absolute value) of the external magnetic field. Interestingly, both scattering lengths achieve asymptotic constant values in the infinitely strong field limit. Remarkably, the observed trends are opposite to the ones predicted as a function of temperature [14], thus suggesting a potential means to experimentally disentangle thermal and magnetic effects. As a natural extension of this work, we are currently examining the combined effect of the thermal and magnetic contributions of the scattering lengths in these isospin channels. Results shall be reported elsewhere.

\section{ACKNOWLEDGMENTS}

M. L. acknowledges support from FONDECYT (Chile) under Grant No. 1170107, 1150471, and 1150847, and ConicytPIA/BASAL (Chile) Grant No. FB0821. L. M. acknowledges support from FONDECYT (Chile) under Grant No. 1170107. A. R. acknowledges support form "Consejo Nacional de Ciencia y Tecnología (Mexico) under Grant No. 256494. R.Z. acknowledges support from CONICYT FONDECYT Iniciación under Grant No. 11160234. E. M. acknowledges support from FONDECYT under Grant No. 1190361.

\section{APPENDIX: INTEGRALS OVER $\boldsymbol{k}_{\|}=\left(k_{0}, k_{3}\right)$}

Here we present in detail the calculation of the integrals involved in Eq. (19) of the main text. Using the definition of the "parallel" propagators (14), we have

$$
\begin{aligned}
G_{l, l}\left(p_{0}\right) & =\iint \frac{d k_{0} d k_{3}}{(2 \pi)^{2}} i \Delta_{l}^{B}\left(k_{0}, k_{3}\right) i \Delta_{l}^{B}\left(k_{0}-2 p_{0}, k_{3}\right) \\
& =\frac{i^{2}}{(2 \pi)^{2}} \int_{-\infty}^{+\infty} d k_{3} f_{l}\left(k_{3}, p_{0}\right)
\end{aligned}
$$

where we have defined the integral

$$
f_{l}\left(k_{3}, p_{0}\right)=\int_{-\infty}^{+\infty} \frac{d k_{0}}{A\left(k_{3}\right) C\left(k_{3}\right)},
$$

with 


$$
\begin{aligned}
& A\left(k_{3}\right)=k_{0}^{2}-E_{l}\left(k_{3}\right)^{2}+i \epsilon, \\
& C\left(k_{3}\right)=\left(k_{0}-2 p_{0}\right)^{2}-E_{l}\left(k_{3}\right)^{2}+i \epsilon,
\end{aligned}
$$

and $E_{l}\left(k_{3}\right)=\sqrt{k_{3}^{2}+m_{\pi}^{2}+q B(2 l+1)}$. The integral can be evaluated in the complex $k_{0}$ plane, by noticing that it possesses four simple poles at $k_{0}= \pm E_{l}\left(k_{3}\right) \mp i \epsilon^{\prime}$ and $k_{0}=2 p_{0} \pm E_{l}\left(k_{3}\right) \mp i \epsilon^{\prime}$, i.e., two located in the positive imaginary plane and two located in the negative imaginary plane. For the integration contour we choose a semicircle, which closes on the upper imaginary plane, and thus it encloses the poles $k_{0}^{(1)}=-E_{l}\left(k_{3}\right)+i \epsilon^{\prime}$ and $k_{0}^{(2)}=2 p_{0}-$ $E_{l}\left(k_{3}\right)+i \epsilon^{\prime}$. By direct application of the residue theorem, we have that

$$
\begin{aligned}
f_{l}\left(k_{3}, p_{0}\right)= & \frac{-2 i \pi}{8 p_{0}}\left[\frac{1}{E_{l}\left(k_{3}\right)\left(E_{l}\left(k_{3}\right)+p_{0}\right)}\right. \\
& \left.-\frac{1}{E_{l}\left(k_{3}\right)\left(E_{l}\left(k_{3}\right)-p_{0}\right)}\right] .
\end{aligned}
$$

Now we calculate the integral over $k_{3}$. Inserting Eq. (A4) into Eq. (A1), we have

$$
G_{l, l}\left(p_{0}\right)=\frac{i}{16 \pi p_{0}}\left(g_{l}\left(p_{0}\right)-g_{l}\left(-p_{0}\right)\right),
$$

where we have defined

$$
\begin{aligned}
g_{l}\left(p_{0}\right) & =\int_{-\infty}^{+\infty} \frac{d k_{3}}{E_{l}\left(k_{3}\right)\left[E_{l}\left(k_{3}\right)-p_{0}\right]} \\
& =2 \int_{0}^{+\infty} \frac{d k_{3}}{E_{l}\left(k_{3}\right)\left[E_{l}\left(k_{3}\right)-p_{0}\right]} \\
& =2 \frac{\frac{\pi}{2}-\operatorname{Arctan}\left(\frac{p_{0}}{\sqrt{q B(2 l+1)+m_{\pi}^{2}-p_{0}^{2}}}\right)}{\sqrt{q B(2 l+1)+m_{\pi}^{2}-p_{0}^{2}}} .
\end{aligned}
$$

Substituting back into Eq. (A5), we obtain the finite result

$$
G_{l, l}\left(p_{0}\right)=\frac{i}{4 \pi p_{0}} \frac{\operatorname{Arctan}\left(\frac{p_{0}}{\sqrt{q B(2 l+1)+m_{\pi}^{2}-p_{0}^{2}}}\right)}{\sqrt{q B(2 l+1)+m_{\pi}^{2}-p_{0}^{2}}} .
$$

Inserting this expression back into Eq. (19) of the main text, we obtain the infinite-series representation

$$
I_{1}\left[B, p_{0}\right]=\frac{i q B}{8 \pi^{2} p_{0}} \sum_{l=0}^{\infty} \frac{\operatorname{Arctan}\left(\frac{p_{0}}{\sqrt{q B(2 l+1)+m_{\pi}^{2}-p_{0}^{2}}}\right)}{\sqrt{q B(2 l+1)+m_{\pi}^{2}-p_{0}^{2}}} .
$$

[1] A. Adare et al. (PHENIX Collaboration), Phys. Rev. C 91, 064904 (2015).

[2] A. Adare et al. (PHENIX Collaboration), Phys. Rev. C 94, 064901 (2016).

[3] J. Adam et al. (ALICE Collaboration), Phys. Lett. B 754, 235 (2016).

[4] A. Ayala, J. D. Castaño-Yepes, C. A. Dominguez, L. A. Hernández, S. Hernández-Ortiz, and M.E. TejedaYeomans, Phys. Rev. D 96, 014023 (2017); 96, 119901 (E) (2017).

[5] For a review see A. Ayala, C. A. Dominguez, and M. Loewe, Adv. High Energy Phys. 2017, 9291623 (2017); C. A. Dominguez, Quantum Chromodynamics Sum Rules, Springer Briefs in Physics (Springer Verlag, Cham, Switzerland, 2018).

[6] V. Kekelidze, A. Kovalenko, R. Lednicky, V. Matveev, I. Meshkov, A. Sorin, and G. Trubnikov, EPJ Web Conf. 182, 02063 (2018).

[7] L. McLerran and R. D. Pisarski, Nucl. Phys. A796, 83 (2007); H. Abuki, R. Anglani, R. Gatto, G. Nardulli, and M. Ruggieri, Phys. Rev. D 78, 034034 (2008).

[8] G. S. Bali, F. Bruckmann, G. Endrodi, Z. Fodor, S. D. Katz, S. Krieg, A. Schafer, and K. Szabo, J. High Energy Phys. 02 (2012) 044; G. S. Bali, F. Bruckmann, G. Endrodi, Z. Fodor, S. D. Katz, and A. Schafer, Phys. Rev. D 86, 071502 (2012).
[9] A. Ayala, M. Loewe, and R. Zamora, Phys. Rev. D 91, 016002 (2015).

[10] A. Ayala, C. A. Dominguez, S. Hernández-Ortiz, L. A. Hernández, M. Loewe, D. Manreza Paret, and R. Zamora, Phys. Rev. D 98, 031501(R) (2018).

[11] X.-H. Liu, F.-K. Guo, and E. Epelbaum, Eur. Phys. J. C 73, 2284 (2013).

[12] M. Loewe, L. Monje, and R. Zamora, Phys. Rev. D 97, 056023 (2018).

[13] M. Loewe and J. Ruiz, Phys. Rev. D 78, 096007 (2008).

[14] M. Loewe and C. Martínez, Phys. Rev. D 77, 105006 (2008); 78, 069902 (2008).

[15] M. Gell-Mann and M. Lévy, Nuovo Cimento 16, 705 (1960).

[16] C. Contreras and M. Loewe, Int. J. Mod. Phys. A 05, 2297 (1990).

[17] A. Larsen, Z. Phys. C 33, 291 (1986).

[18] N. Bilic and H.Nikolic, Eur. Phys. J. C 6, 513 (1999).

[19] H. Mao, N. Petropoulos, and W-K. Zhao, J. Phys. G 32, 2187 (2006); N. Petropoulos, arXiv:hep-ph/0402136 and references therein.

[20] B. J. Schaefer and M. Wagner, Phys. Rev. D 79, 014018 (2009).

[21] P. Kovacs and Z. Szep, Phys. Rev. D 77, 065016 (2008).

[22] P. Kovacs and Z. Szep, Phys. Rev. D 75, 025015 (2007). 
[23] P. Kovacs and Z. Szep, Phys. Rev. D 93, 114014 (2016).

[24] P. D. B. Collins, An Introduction to Regge Theory on High Energy Physics (Cambridge University Press, Cambridge, England, 1977).

[25] J. Gasser and H. Leutwyler, Ann. Phys. (N.Y.) 158, 142 (1984).
[26] L. Rosellet et al. Phys. Rev. D 15, 574 (1977).

[27] B. Adeva et al. Phys. Lett. B 704, 24 (2011).

[28] X.-H. Liu, F.-K. Guo, and E. Epelbaum, Eur. Phys. J. C 73, 2284 (2013).

[29] A. Ayala, A. Sanchez, G. Piccinelli, and S. Sahu, Phys. Rev. D 71, 023004 (2005).

[30] B. Peyaud, Nucl. Phys. B, Proc. Suppl. 187, 29 (2009). 